\title{
DÉSIGNER LE SCANDALE. LE PARATEXTE DES PAMPHLETS CATHOLIQUES ENTRE ACCUSATION ET LÉGITIMATION (FRANCE ET PAYS-BAS HABSBOURGEOIS, 1579-1590)*
}

\begin{abstract}
Alexandre Goderniaux
En septembre 1589, le Parlement de Paris publie un arrêt interdisant «à tous imprimeurs, libraires et colporteurs d'imprimer, vendre ni exposer en vente aucuns libelles scandaleux et diffamatoires et généralement d'imprimer aucuns livres et petits livrets sans permission de ladite cour ou du juge ordinaire ${ }^{1}$ ». Avec « diffamatoire », " scandaleux » est l'épithète la plus régulièrement employée pour dénoncer les courts textes polémiques produits durant les guerres de Religion en France et dans les Pays-Bas habsbourgeois². Or, tandis que plusieurs libelles élaborent des stratégies pour éloigner d'eux cette accusation de scandale, nombre d'entre eux exploitent cette notion pour accuser ou argumenter. La publicité du scandale est en effet le plus incontesté de ses traits définitoires ${ }^{3}$ : cet « acte de langage ${ }^{4}$ » a besoin $d^{\prime}$ « instruments de mise en visibilité ${ }^{~} \gg$ et le libelle, dont la fonction essentielle est de commenter voire de révéler des faits dont la connaissance est traditionnellement réservée à la seule élite gouvernante, est, durant les guerres de Religion, l'outil de publication du scandale par excellence. C'est pourquoi, tout en cherchant à l'éloigner, il fabrique le scandale.
\end{abstract}

* Nous souhaitons adresser nos sincères remerciements à Blandine Perona, grâce à qui cette enquête a pu être publiée dans ce volume, et dont les relectures attentives ont permis d'affiner nos analyses.

$1 \quad$ Arrest de la cour de Parlement pour la conservation du repos public de la ville et fauxbourgs de Paris, cité par Denis Pallier, Recherches sur l'imprimerie à Paris pendant la Ligue (1585-1594), Genève, Droz, 1975, p. 71-72. Nos italiques. Sur la législation anti-libelles durant les guerres de Religion, voir ibid., p. 55 ; Tatiana Debbagi Baranova, "Les guerres de Religion : guerres de médisance ? », dans Sylvie Mougin (dir.), La Médisance. Actes du colloque international de Reims Champagne-Ardenne, les 25, 26 et 27 septembre 2003, Reims, Presses universitaires de Reims, 2006, p. 472.

2 Tatiana Debbagi Baranova, À coups de libelles. Une culture politique au temps des guerres de Religion (1562-1598), Genève, Droz, 2012, p. 35-36.

3 Cyril Lemieux et Damien de Blic, «Le scandale comme épreuve. Éléments de sociologie pragmatique », Politix, 71, 2005, p.14-15, [En ligne] https://www.cairn.info/revue-politix-2005-3-page-9.htm [consulté le 28/10/2021] ; Sylvain Parasie, " Des machines à scandale. Éléments pour une sociologie morale des bases de données », Réseaux, 2, 2013, p. 129, [En ligne] https://www.cairn.info/revue-reseaux2013-2-page-127.htm [consulté le 28/10/2021] ; Mathilde Bernard, « La conversion de Pierre-Victor Palma Cayet ou le règne du scandale », dans Cécile Huchard et Jean-Claude Ternaux (dir.), Calliope et Mnémosyne. Mélanges offerts à Gilbert Schrenck, Paris, Classiques Garnier, 2017, p. 158 ; Anne-Pascale Pouey-Mounou, Panurge comme lard en pois. Paradoxe, scandale et propriété dans le Tiers Livre, Genève, Droz, 2013, p.71; Élise Monjarret, « Donnerà voir et conjurer le scandale: le pamphlet comme rhétorique de l'événement contre l'événement », dans Christiane Rivalan Guégo et Denis Rodrigues (dir.), L'Écho de l'événement. Du Moyen Âge à l'époque contemporaine, Rennes, Presses universitaires de Rennes, 2011, p. 167-175, [En ligne] https://books.openedition.org/pur/40788\#bodyftn2 [consulté le 28/10/2021].

4 John B. Thompson cité et traduit par Cyril Lemieux et Damien de Blic, « Le scandale comme épreuve », art. cit., p. 15.

5 Sylvain Parasie, « Des machines à scandale », art. cit., p. 129. 
Cet article propose d'expliquer cette apparente contradiction dans le rapport du libelle au scandale en étudiant son paratexte. Les travaux pionniers de Gérard Genette ont montré que ce lieu où les stratégies rhétoriques sont concentrées constitue un excellent observatoire des intentions de l'auteur de l'œuvre tout entière ${ }^{6}$. Grand fournisseur de clefs de lecture, le paratexte est l'emplacement par excellence où le sens et le dispositif de la publication sont explicités et où l'auteur noue les liens entre le texte et le contexte ${ }^{7}$. Cette enquête envisagera dès lors les pièces liminaires et les notes marginales des libelles comme des métadiscours où leur portée et leur fonctionnement discursif, souvent très implicites, apparaissent avec clarté. Pièces liminaires et notes marginales fonctionnant en système au sein des libelles, elles seront interrogées à travers le prisme de leur double fonction de légitimation et d'accusation contre et par la notion de scandale ${ }^{8}$.

Cet article étudiera dix libelles sélectionnés pour la richesse des fonctions et des stratégies argumentatives de leur paratexte. Ces sources sont issues de deux phases différentes des guerres de Religion. D'une part, la Ligue parisienne qui, de 1585 à 1594, s'oppose à l'accession au trône du protestant Henri de Navarre ${ }^{9}$ et publie une telle quantité de libelles qu'elle établit un record de production imprimée ${ }^{10}$. D'autre part, dans les dix-sept provinces constituant les Pays-Bas, la répression intransigeante du protestantisme et la centralisation du gouvernement par le roi Philippe II conduisent à un soulèvement mené par Guillaume d'Orange et au ralliement de plusieurs villes et provinces aux rebelles. Dès 1578, le gouverneur général Alexandre Farnèse parvient à reconquérir progressivement une partie de ces territoires par diverses initiatives militaires et diplomatiques" ${ }^{11}$. Des textes polémiques sont alors imprimés pour soutenir par les mots cette entreprise de reconquête ${ }^{12}$. Produits dans des contextes et des espaces géographiques différents, les libelles de la Ligue parisienne et les textes polémiques favorables

6 Gérard Genette, Seuils, Paris, Seuil, 2002.

7 Patrick Marot, «Pour une poétique historique des textes liminaires », dans id. (dir.), Les Textes liminaires, Toulouse, Presses universitaires du Mirail-Toulouse, 2010, p. 7-27.

8 Le scandale est donc envisagé ici dans une optique stratégiste, étudiant les « stratégies scandaleuses » ou "stratégies de scandalisation » (Michel Offerlé cité par Cyril Lemieux et Damien de Blic, «Le scandale comme épreuve », art. cit., p. 22). Cette lecture du scandale n'est pas exempte de limites (voir ibid., p. 25-27; Hervé Rayner, Fabien Thétaz et Bernard Voutat, «L'indignation est-elle un ressort de la scandalisation? Le "scandale des fiches" en Suisse », Éthique et scandales publics, 18, 2016, [En ligne] https://journals.openedition.org/ethiquepublique/2847 [consulté le 28/10/2021]). Loin d'ambitionner de poser un point final à la réflexion, cette enquête se conçoit comme une première étape de l'étude du scandale dans les textes retenus : ses résultats doivent être considérés comme le point de départ d'autres lectures à même de les affiner.

9 Nicolas Le Roux, Un régicide au nom de Dieu. L'assassinat d'Henri III (1ªr août 1589), Paris, Gallimard, 2006 ; Jean-Marie Constant, La Ligue, Paris, Fayard, 1996.

10 Denis Pallier, Recherches sur l'imprimerie, op. cit.

11 Violet Soen, «Philippe II et les anciens Pays-Bas. Les limites d'un gouvernement à distance dans un empire global (1555-1598) », Histoire, Économie et Société, 38, 2019, p. 11-32, [En ligne] https:// www.cairn.info/revue-histoire-economie-et-societe-2019-3-page-11.htm [consulté le 28/10/2021] ; Ead., "Reconquista and Reconciliation in the Dutch Revolt. The campaign of Governor-General Alexander Farnese (1578-1592) », Journal of Early Modern History, 16, 2012, p. 1-22 ; Yves Junot, «Pratiques et limites de la réconciliation après les guerres de Religion dans les villes des Pays-Bas méridionaux (années 1570-années 1590) », Revue du Nord, 395, 2012, p. 327-346, [En ligne] https:// www.cairn.info/revue-du-nord-2012-2-page-327.htm [consulté le 28/10/2021].

12 Monica Stensland, Habsburg communication in the Dutch revolt, Amsterdam, Amsterdam University Press, 2012, p. 89-100; Alexander Soetaert, «Printing at the frontier. The emergence of a transregional book production in the Ecclesiastical Province of Cambrai (ca 1560-1659) », De Gulden Passer, 94, 2016, p. 137-163; Id., Violet Soen et Johan Verberckmoes, « Verborgen meertaligheid. De katholieke drukpers in de kerkprovincie Kamerijk (1560-1600) », Queeste, 22, 2015, p. 62-81 ; Christiane Piérard et Pierre Ruelle, Les Premiers livres imprimés à Mons, Mons, Société des bibliophiles belges, 1966. 
aux Habsbourg publiés sous le gouvernorat d'Alexandre Farnèse présentent toutefois de nombreuses caractéristiques communes : longtemps négligés voire dénigrés, ils forment un corpus catholique cohérent que les chercheurs reconsidèrent depuis quelques années ${ }^{13}$.

La première fonction du paratexte consiste à légitimer l'acte même de diffusion d'un texte polémique. La nouveauté que constitue le libelle explique l'accusation de scandale qui le frappe régulièrement: durant les guerres de Religion, la démultiplication inédite de l'expression, publique ou non, de commentaires politiques est régulièrement critiquée par des auteurs qui en font le symptôme voire la cause d'un dérèglement de la société ${ }^{14}$. Ceci explique le glissement du sens du scandale au milieu du XVI siècle : jusqu'alors entendu principalement dans sa définition théologique d'occasion ou de cause du péché, le terme « scandale » est aussi utilisé pour désigner des paroles ou des actes qui provoquent le désordre ou menacent l'équilibre de l'État. C'est dans ces deux acceptions que le scandale est une accusation régulièrement employée dans le cadre des affrontements entre catholiques et protestants ${ }^{15}$. Dès lors, les libelles constituent des « lieux de scandale ${ }^{16}$ » à double titre : le scandale au sens évangélique a depuis toujours besoin d'« espaces de "scandalisation"17 » publics, mais la démultiplication de ces derniers par l'imprimé polémique et la généralisation du « mésusage de la parole ${ }^{18}$ » par les libelles constituent ellesmêmes un scandale, au sens politique. Ainsi, souvent, au XVIe siècle, le libelle incarne le scandale : dans leur dénonciation des paroles provoquant le désordre, les différents camps emploient le terme « libelle » comme une insulte pour discréditer les écrits de leurs adversaires ${ }^{19}$. La nature scandaleuse des libelles, qui fascine autant qu'elle attise les critiques, apparaît ainsi comme un facteur décisif de la mutation de l'espace public du second XVle siècle ${ }^{20}$.

13 Marco Penzi, « Damnatio memoriae. La Ligue Catholique française e la storiografia, tra politiques, rivoluzionari, mistici e liberali », Quaderni storici, 40, 2005, p. 263-284, [En ligne] https://www. jstor.org/stable/43779483?seq=1\#metadata info tab_contents [consulté le 28/10/2021] ; Monica Stensland, Habsburg communication in the Dutch revolt, op. cit.

14 Nicolas Le Roux, "Il est impossible de tenir les langues des hommes bridées". Opinion commune et autorité monarchique au temps des troubles de religion », dans Laurent Bourquin et al. (dir.), S'exprimer en temps de troubles. Conflits, opinion(s) et politisation de la fin du Moyen Âge au début du $X X^{e}$ siècle, Rennes, Presses Universitaires de Rennes, 2011, p. 63.

15 Sur ces glissements de sens, voir la mise au point lexicographique dans l'introduction de cet ouvrage. Sur l'évolution de la définition du scandale au XVI siècle, voir aussi Mathilde Bernard, «La conversion de Pierre-Victor Palma Cayet », art. cit., p. 169 ; Stéphane Van Damme, « L'éternel retour du scandale? », Hypothèses, 16, 2013, p. 230-231, [En ligne] https://www.cairn.info/revuehypotheses-2013-1-page-227.htm [consulté le 28/10/2021] ; Anne-Pascale Pouey-Mounou, Panurge comme lard en pois, op. cit.

16 Anne-Pascale Pouey-Mounou, «Calvin et le scandale », dans Franco Giacone (dir.), Calvin insolite, Paris, Classiques Garnier, 2012, p. 89.

17 Émilie Dosquet et François-Xavier Petit, « Faire scandale. Éléments de définition, enjeux méthodologiques et approches historiographiques », Hypothèses, 16, 2013, p. 149, [En ligne] https:// www.cairn.info/revue-hypotheses-2013-1-page-147.htm [consulté le 28/10/2021].

18 Anne-Pascale Pouey-Mounou, "Calvin et le scandale », art. cit., p. 89. Au XVI siècle, ne pas garder le secret ou critiquer des absents est considéré comme un détournement de la parole (Tatiana Debbagi Baranova, «Les guerres de Religion : guerres de médisance?», art. cit., p. 467). Sur les liens entre médisance et scandale, voir Max Gluckman, " Gossip and Scandal », Current Anthropology, 4, 1963, p. 307-316, [En ligne] https://www.jstor.org/stable/2739613?origin=/STOR-pdf\&seq=1\#metadata info tab contents [consulté le 28/10/2021].

19 Tatiana Debbagi Baranova, À coups de libelles, op. cit., p. 35-36.

20 Diane Roussel, « L'espace public comme enjeu des guerres de Religion et de la paix civile. Réflexions sur la notion d'espace public et ses métamorphoses à Paris au XVI siècle », dans Patrick Boucheron et Nicolas Offenstadt (dir.), L'Espace public au Moyen Âge. Débats autour de Jürgen Habermas, Paris, PUF, 2011, p. 131-146, [En ligne] https://www.cairn.info/l-espace-public-au-moyen-age--9782130573579page-131.htm [consulté le 28/10/2021]. 
Par conséquent, légitimer la publication d'un libelle consiste à l'éloigner de l'accusation de scandale dans son acceptation politique. À cette fin, les polémistes utilisent fréquemment la technique de la « publication » de texte : un document (totalement apocryphe) est parvenu à l'imprimeur dans d'étranges circonstances et se révèle tellement éclairant à propos des débats politiques en cours que celui-ci décide de l'imprimer. Ces publications sont parfois précédées d'une pièce préliminaire où cet historique de composition du texte - probablement créé de toute pièce - est détaillé. Le Discours veritable du martyre de deux prebstres et deux laycs est ainsi introduit par un avis de "l'imprimeur au lecteur » où apparaît clairement cet effort de contournement de l'accusation de scandale par la narration des péripéties de la publication :

M'estant tombé entre les mains ceste presente histoire d'aucuns catholiques martyrisez en Angleterre pour la deffense de la foy catholique, j'ay jugé qu'il n'estait point hors de saison, apres la publication du Jubilé concedé de sa Saincteté pour les necessitez de la France, de la mettre en lumiere $[\ldots]^{21}$.

Une des variantes les plus fréquentes de cette technique de « publication » de texte consiste à diffuser des documents préjudiciables pour l'adversaire, présentés comme produits par ce dernier et miraculeusement interceptés : la source " est publiée comme par hasard, en mettant au jour des informations secrètes ${ }^{22}$ ", voire des aveux très compromettants ${ }^{23}$. Dans les Pays-Bas, les catholiques impriment ainsi une Lettre intercepte du prince d'Oranges au duc d'Alençon dont l'avis « au lecteur » explique que « Dieu a miraculeusement permis qu'elle vint en nos mains pour par tout le monde publier, \& faire cognoistre son impieté24 ». De telles constructions discursives s'observent en France également. La Ligue parisienne " publie » ainsi une Lettre escrite à madame de Tinteville dont l'avis au lecteur indique : "Amy lecteur, m'estant tombé entre mains ceste missive [...], il m'a semblé qu'il ne seroit hors de propos de la mettre sur la presse ${ }^{25}$. »Quelques lignes plus bas, le même texte liminaire précise : "Si vous doutés [sic] que ce soit chose supposee, l'imprimeur vous pourra donner certaines nouvelles de l'original ${ }^{26}$. » Cette méticulosité dans l'authentification du document atteste le besoin d'accréditation et de légitimation du discours libelliste dans l'espace public des guerres de Religion.

La révélation au public des documents interceptés serait un scandale s'ils n'étaient pas authentiques : l'opération de légitimation qui se joue dans les pièces préliminaires est essentielle pour prémunir le libelle contre cette accusation. À des fins d'accréditation, le texte liminaire crée une situation d'élocution et fournit les éléments à même de l'attester. Le paratexte,

21 Discours veritable du martyre de deux prebstres \& deux laycs, Paris, Guillaume Chaudière, 1590, p. 3, [En ligne] https://gallica.bnf.fr/ark:/12148/bpt6k526422.image [consulté le 28/10/2021].

22 Tatiana Debbagi Baranova, À coups de libelles, op. cit., p. 440.

23 Pieter Geurts, De Nederlandse Opstand in de pamfletten 1566-1584, Nimègue, Centrale Drukkerij, 1956, p. 61-65, 70, 78, 83-88, 98-107, [En ligne] https://www.dbnl.org/tekst/geur004nede01_01/, consulté le 28/10/2021 ; Alexandre Goderniaux, " "Leurs propos le tesmoignent assez." Le discours rapporté par voie épistolaire, arme de la Ligue parisienne (1585-1594) », Karine Abiven et Cécile Lignereux (dir.), Les Discours rapportés en contexte épistolaire (XVIe-XVIII siècle), Dossier Acta Litt\&Arts, 13, 2020, [En ligne] http://ouvroir-litt-arts.univ-grenoble-alpes.fr/revues/actalittarts/602--leurspropos-le-tesmoignent-assez-le-discours-rapporte-par-voie-epistolaire-arme-de-la-ligueparisienne-1585-1594 [consulté le 28/10/2021].

24 Lettre intercepte du prince d'Oranges au duc d'Alençon. Avecq quelques avdertissements sur icelle pour ouvrir les yeulx aux bons subjects, s.l., s.n., 1580, p. [3], [En ligne] https://lib.ugent.be/catalog/ rug01:002212269 [consulté le 28/10/2021].

25 Lettre escrite à madame de Tinteville, Paris, Guillaume Chaudière, 1589, p. 3, [En ligne] https:// books.google.be/books?id=FwdmAAAAcAAJ\&printsec=frontcover\&hl=fr\#v=onepage\&q\&f=false [consulté le 28/10/2021].

26 Ibid., p. 4. 
outil traditionnel de justification de la publication ${ }^{27}$, est ainsi le lieu où se construit la norme discursive de tout le libelle. Il est aussi celui où se joue la première étape de la diffamation de l'ennemi : l'avis au lecteur éloigne l'accusation de scandale - au sens politique - du libelle et la renvoie - au sens évangélique - dans le camp adverse. À cette fin, il exploite un élément constitutif de l'essence même du libelle : la vérité28.

Autolégitimation et accusation de l'adversaire se rejoignent quand l'avis au lecteur indique que le texte introduit est publié « parce qu'en ce temps calamiteux auquel nous sommes, plusieurs espritz remuantz ne cessent de calumnier les conseilz \& actions des gens de bien, semantz à c'est [sic] effect plusieurs libelz \& faulx raportz ${ }^{29} »$. Ce contexte de désinformation permet de proclamer la nécessité de publier la véritable version des faits : le texte-seuil du même libelle affirme que la publication introduite est conçue « pour fermer la bouche aux detracteurs ${ }^{30}$ » et, surtout, " afin que telles premieres opinions ne prennent trop avant racine aux cerveaux des hommes, au prejudice de la verité ${ }^{31} »$. C'est avant tout la rhétorique judiciaire qui guide la construction des avis au lecteur des libelles, dont les auteurs sont souvent des juristes ${ }^{32}$. La revendication de la vérité est cruciale dans leur accusation, de même que la condamnation antithétique du mensonge de leurs adversaires. L'avis au lecteur d'un autre libelle affirme, par exemple, que le texte qui le suit présente les faits « sans fard \& desguisement de langage, ains avec une pure \& simple verité33 ». L'exposé des raisons de la publication présente précisément celle-ci comme un combat en faveur de la vérité : par cette argumentation, il construit un ethos intègre pour le locuteur, légitime la diffusion du libelle et accuse ses ennemis de mensonge. Ce faisant, la pièce liminaire prouve que, loin de constituer un scandale - au sens politique -, la publication du libelle est, au contraire, revendiquée comme indispensable pour lutter contre le scandale - au sens évangélique - : le critère de vérité légitime autant qu'il accuse.

D'après le paratexte des libelles catholiques, les publications mensongères de leurs adversaires ne sont que le reflet de leurs fallacieux desseins. Un avis au lecteur accuse ainsi les ennemis des ligueurs de tenir des « discours fardez [...] ausquels a esté besoing de respondre [...] pour descouvrir le masque de ces pippeurs ${ }^{34} »$. Les pièces liminaires des publications de documents interceptés exploitent tout particulièrement cette rhétorique : la Lettre escrite à madame de Tinteville débute par un avis « au zelé et catholique lecteur » qui annonce mettre la

27 Yohann Deguin et Marine Roussillon (dir.), Depuis les marges : le pouvoir dans le livre aux $X V l^{e}$ et XVIIe siècles (à paraître en 2022). Nous remercions Yohann Deguin pour nous avoir permis d'accéder aux manuscrits de cette publication.

28 Tatiana Debbagi Baranova, « La vérité et les stratégies d'accréditation du discours politique pendant les guerres de Religion en France », dans Jean-Philippe Genet (dir.), La Vérité. Vérité et crédibilité : construire la vérité dans le système de communication de l'Occident (XIII'-XVII siècle), Paris-Rome, Éditions de la Sorbonne/École française de Rome, 2017, [En ligne] https://books.openedition.org/ psorbonne/6652?lang=fr [consulté le 28/10/2021].

29 Recueil des lettres, actes et pieces plus signalees du progres et besongne faict en la ville d'Arras \& ailleurs, Douai, Jean Bogard, 1579, fol. A2r. Nos italiques, [En ligne] https://gallica.bnf.fr/ark:/12148/ bpt6k65158623.textelmage [consulté le 28/10/2021].

30 Ibid., fol. A3v.

31 Ibid., fol. A2r-v.

32 Tatiana Debbagi Baranova, «La vérité et les stratégies d'accréditation du discours », art. cit. ; Denis Pallier, Recherches sur l'imprimerie, op. cit., p. 134 et 460-465.

33 Discours veritable touchant plusieurs affaires d'Estat, pour la justification des bons et fideles subjectz de Sa Majesté catholique, Douai, Jean Bogard, 1580, fol. 3r, [En ligne] https://books.google.be/books?id= qrnHEWtO9UwC\&printsec=frontcover\&hl=fr\#v=onepage\&q\&f=false [consulté le 28/10/2021].

34 Memoires semez par quelques politics aux Estats qui se tiennent en la ville de Bloys. Avec la response catholique à iceux, Paris, s.n., 1588, p. 6, [En ligne] https://gallica.bnf.fr/ark:/12148/bpt6k79565j [consulté le 28/10/2021]. 
lettre « sur la presse afin que chacun descouvrant la ruze de ces regnards se donnast garde plus aisément de leurs surprises ${ }^{35} »$. La métaphore du dévoilement est encore plus explicite dans le titre alternatif sous lequel la même lettre a été imprimée : Le Masque descouvert du Biernois et ses adhérents, par une lettre envoyee à madame de Tinteville ${ }^{36}$. La vérité permet aux libellistes de recourir, dans le même avis au lecteur, aux rhétoriques judiciaire et épidictique, via un blâme renforçant l'accusation. Cette dénonciation de la feintise et de la cautèle de l'adversaire, topos du discours satirique fréquemment utilisé par les libellistes catholiques ${ }^{37}$, permet aux pièces liminaires de retourner l'arme du scandale contre l'adversaire : l'exhibition de faits secrets par un document dont la découverte est théâtralisée construit un « drame de la dissimulation et du dévoilement ${ }^{38} »$. Le scandale porte atteinte à la réputation et l'honneur des cibles de ce procédé ${ }^{39}$. Le libelle ne se contente pas de sélectionner un élément d'apparence anecdotique et révélateur d'enjeux plus larges : il exploite sa révélation au public pour mettre en place une spectacularisation des événements rapportés ${ }^{40}$, propre à susciter l'indignation, moteur du scandale.

Les liens entre le scandale et l'indignation, extrêmement anciens ${ }^{41}$, se reconfigurent durant la première Modernité ${ }^{42}$. Le scandale « exige une certaine unanimité dans l'indignation ${ }^{43}$ », tout particulièrement quand il est utilisé au sein d'une stratégie discursive ${ }^{44}$ : employé comme une arme juridique dès le Moyen Âge ${ }^{45}$, il devient une arme juridico-politique au XVle siècle ${ }^{46}$. Les textes interceptés et publiés, au cœur de cette enquête, constituent le lieu idéal pour nouer des liens entre indignation et scandale : « la question des montages, d'objets et de documents, est d'ailleurs souvent ce qui fait scandale, ce qui produit l'indignation ${ }^{47} \gg$.

35 Lettre escrite à madame de Tinteville, op. cit., p. 3.

36 Denis Pallier, Recherches sur l'imprimerie, op. cit., p. 341.

37 Alexandre Goderniaux, "Le "voile commun à tous meschans". La justification de l'intolérance par la rhétorique du dévoilement dans la polémique catholique (France et Pays-Bas habsbourgeois, 1580-1594) », Loxias, 18, 2021, [En ligne] http://revel.unice.fr/symposia/actel/index.html?id=1822 [consulté le 16/12/2021] ; Camille Dohet, «Prudence, cautèle et dissimulation : le double discours des élites politiques durant la guerre de Quatre-Vingts ans », Revue belge de Philologie et d'Histoire, 94, 2016, p. 883-920.

38 John B. Thompson cité et traduit par Cyril Lemieux et Damien de Blic, « Le scandale comme épreuve », art. cit., p. 21.

39 Journée d'étude du 8 février 2019 du projet Mémoire et Usages des récits et images du Mal (compte-rendu en ligne), [En ligne] https://mum.hypotheses.org/747\#more-747 [consulté le 28/10/2021]).

40 Ruth Amossy, Apologie de la polémique, Paris, PUF, 2014, p. 48-49, 69, 82-83, 110-112, 116-117, 212.

41 Voir, à ce sujet, Marion Gauthier, Scandale et indignation dans les sermons de Bossuet, Bourdaloue et Massillon, thèse inédite, Universités Sorbonne Paris Cité et Paris Diderot, 2017, p. 12-47, [En ligne] http://theses.md.univ-paris-diderot.fr/GAUTHIER Marion 2 va 20170915.pdf [consulté le 28/10/2021].

42 Ibid., p. 269-301.

43 Nathalie Heinich, "L'art du scandale. Indignation esthétique et sociologie des valeurs », Politix, 71, 2005, p. 125, [En ligne] https://www.cairn.info/revue-politix-2005-3-page-121.htm [consulté le 28/10/2021].

44 Cyril Lemieux et Damien de Blic, « Le scandale comme épreuve », art. cit., p. 22-23. " Caractéristique du discours polémique » (Ruth Amossy, Apologie de la polémique, op. cit., p. 153), elle est aussi « une arme politique » (Ibid., p. 173). Cette lecture stratégiste doit, ici aussi, être nuancée : l'indignation constitue une arme efficace quand le groupe qui l'exprime est uni par des valeurs communes (Hervé Rayner, Fabien Thétaz et Bernard Voutat, " L'indignation est-elle un ressort de la scandalisation? », art. cit.).

45 Arnaud-Vivien Fossier, « Propter vitandum scandalum. Histoire d'une catégorie juridique (XII"-XVe siècle) », Mélanges de l'École française de Rome, 121, 2009, p. 317-348.

46 Voir l'article de Solange Ségala dans le présent ouvrage.

47 Nicolas Offenstadt et Stéphane Van Damme, «Introduction », dans Luc Boltanski et al. (dir.), Affaires, scandales et grandes causes. De Socrate à Pinochet, Paris, Stock, 2007, p. 14-15. 
L'indignation est le moteur de la seconde fonction des pièces préliminaires, fonction que celles-ci partagent d'ailleurs avec les marginalia : fournir une interprétation du libelle. $\mathrm{Si}$ « assurer au texte une bonne lecture ${ }^{48}$ » est un objectif classique de la préface, l'avis au lecteur des publications polémiques s'inscrit aux antipodes du style généralement encomiastique du texte d'escorte ${ }^{49}$ et utilise cette fonction didactique pour construire l'accusation de l'adversaire. Le libelle intitulé Trahison descouverte de Henry de Valois débute ainsi par ce quatrain en alexandrins:

Lecteur ly ce discours demonstrant les menees

De Henry de Valois fauteur de tous meschans

Perfide, desloyal, hay des bonnes gens

Et trouveras que Dieu n'ayme ses destinees ${ }^{50}$.

Cette introduction versifiée recourt à la rhétorique épidictique pour amplifier les vices de l'adversaire, susciter l'indignation et, partant, fabriquer le scandale. Les propos liminaires font plus qu'identifier une seule manière de lire ce qui suit : ils rejettent toute possibilité de contradiction. Ainsi, l'« advertissement au lecteur » d'une publication pro-Habsbourg parue à Douai en 1579 indique que « quiconque vouldra se despouiller de toute passion, percevra aisment [sic] [...] la droicte \& saincte intention desdictes provinces ${ }^{51} \gg$. Tout qui lirait le libelle autrement que selon l'interprétation énoncée par le texte d'escorte ne serait pas un bon lecteur. Les préambules polémiques revêtent donc une fonction normative.

Toutefois, le paratexte prend soin de dissimuler ce cadrage de l'interprétation. Les pièces liminaires oscillent en effet entre deux logiques en apparence contradictoires. D'une part, la publication de documents s'appuie sur ce que Tatiana Debbagi Baranova identifie comme une éloquence de type judiciaire, " qui doit être, en principe, sobre, informative et fonctionnelle, appelant à la raison et aux faits ${ }^{52} »$. Or cette rhétorique correspond à une posture raisonnée de l'auteur, qui reconnaît au lecteur « une capacité d'argumenter et l'invite à juger de la validité de sa démonstration ${ }^{53} »$. Si certaines pièces liminaires suivent cette logique, telle celle de la Lettre escrite à madame de Tinteville se concluant par « Je vous laisse juge par ceste lecture ${ }^{54}$ », d'autres accompagnent de manière appuyée le lecteur en ne lui laissant qu'une seule interprétation possible de la publication. Alors que la rhétorique judiciaire « consiste à faire croire au lecteur que la vérité se dégage d'elle-même de la présentation brute et dépassionnée des documents et des faits ${ }^{55} »$, la dimension didactique du paratexte illustre que, pour fabriquer un scandale - au sens évangélique -, il ne suffit pas de laisser la vérité se dégager « d'elle-même » : il faut l'expliciter, la reconstituer à partir des faits présentés. Plus que jamais, la vérité est brandie comme une arme par les libellistes cherchant à dissimuler le fait que leurs textes sont des créations formulant accusations et interprétations partisanes. Cette opération de reconstruction de la " vérité » pourrait dès lors être elle-même qualifiée de scandaleuse - au sens politique - par les détracteurs des libelles. C'est pour cette raison qu'elle a lieu en marge du texte. 28/10/2021].

51 Recueil des lettres, actes et pieces plus signalees, op. cit., f. A3v.

52 Tatiana Debbagi Baranova, À coups de libelles, op. cit., p. 452-453.

53 Ibid., p. 447.

54 Lettre escrite à madame de Tinteville, op. cit., p. 4.

55 Tatiana Debbagi Baranova, « La vérité et les stratégies d'accréditation du discours », art. cit. 
Le paratexte est indispensable au corps car lui seul peut faire aboutir le processus de fabrication du scandale, mais le corps est indispensable au paratexte car lui seul peut fournir la matière de cette opération polémique. Cette indivisibilité de la répartition des tâches s'observe clairement dans le recueil de documents intitulé Metamorphose d'Henry de Bourbon jadis roy de Navarre. La source débute par un avis de "l'imprimeur au vray \& naturel François » au sein duquel Jean Pillehotte, imprimeur de la Ligue à Lyon, attaque la volonté du futur Henri IV d'obtenir l'absolution du pape : «Il n'aura point d'honte de feindre d'estre catholique, \& se vouloir encores un coup reconcilier à l'Eglise. J'ay bien voulu remettre en memoire au monde l'essay qu'il en a desja fai [sic] une fois ${ }^{56}$. „ Cette remise en mémoire prend la forme d'une reproduction des pièces relatives à la première conversion du roi de Navarre et à son relaps déclaré par Sixte Quint. Comme d'autres recueils de documents imprimés durant les guerres de Religion, la Metamorphose d'Henry de Bourbon confronte des textes des deux camps : les lettres où le roi de Navarre proclame la sincérité de sa conversion côtoient la bulle pontificale qui l'excommunie.

La plupart du temps, les libelles-recueils présentent les documents sans plus de commentaires : les pièces sélectionnées sont d'une force argumentative tellement inégale que le parti des concepteurs ne peut que l'emporter ${ }^{57}$. Or, à nouveau, le paratexte intervient pour ne pas laisser l'interprétation au hasard et effectuer un travail d'explicitation et de construction. Seul l'avis au lecteur permet de comprendre la logique de la sélection des textes et le titre du recueil : si la publication est intitulée Metamorphose d'Henry de Bourbon, c'est parce que la compilation et la confrontation des documents, conçues selon une " stratégie de montage textuel ${ }^{58}$ ", permettent de prouver que le prétendant au trône de France est versatile. L'avis au lecteur mentionne clairement que cette inconstance est la conséquence de l'opportunisme d'Henri de Bourbon, « qui change de couleur selon la pierre à laquelle il s'attache ${ }^{59}$ ». La signification de toute la publication y est explicitement résumée en quelques mots : le prétendant au trône est accusé de «feindre d'estre catholique ». Si le texte constitue la preuve, c'est dans le paratexte que le libelliste (en l'occurrence, l'imprimeur) accuse et donne son sens à toute la publication.

Traditionnellement lieu d'éloge du pouvoir ${ }^{60}$, le paratexte peut ainsi constituer une tribune pour accuser voire diffamer. Rendre le scandale public est aussi indispensable que complexe : la divulgation de faits ne suffit pas, elle doit être accompagnée de l'explication - qui consiste toujours en une construction - de leur signification ${ }^{61}$. La logique juridique qui traverse les libelles des guerres de Religion permet de comprendre le rôle indissociable des deux parties du libelle. Dans le corps du texte se succèdent les témoignages. Ils sont indispensables à la validité de la démonstration mais ne suffisent pas à attester la nature scandaleuse des faits soumis au jugement du lecteur. Le paratexte est le réquisitoire, le lieu où se nouent tous les fils du corps du texte, la conclusion par anticipation, le coup fatal porté à l'adversaire.

56 Metamorphose d'Henry de Bourbon jadis roy de Navarre, Lyon, Jean Pillehotte, 1589, p. 4, [En ligne] https://gallica.bnf.fr/ark:/12148/bpt6k79652g.image [consulté le 28/10/2021].

57 Cécile Huchard, " Des Mémoires de Condé aux Mémoires de l'Estat de France de Goulart. Le rôle des compilations pamphlétaires », dans Jacques Berchtold et Marie-Madeleine Fragonard (dir.), La Mémoire des guerres de religion. La concurrence des genres historiques (XVIe-XVIII siècle), Genève, Droz, 2006, p. 88-106; Tatiana Debbagi Baranova, "La vérité et les stratégies d'accréditation du discours », art. cit.

58 Tatiana Debbagi Baranova, À coups de libelles, op. cit., p. 458.

59 Metamorphose d'Henry de Bourbon, op. cit., p. 3.

60 Yohann Deguin et Marine Roussillon (dir.), Depuis les marges, op. cit.

61 Sylvain Parasie, « Des machines à scandale », art. cit., p. 129. 
La pièce liminaire ne se contente pas d'énoncer une interprétation incontestable du texte qu'il introduit : elle indique comment agir après l'avoir lu. D'après Gérard Genette, la préface dit : « Voici pourquoi et voici comment vous devez lire ce livre ${ }^{62}$. » Liant intimement les deux questions - pourquoi et comment -, les avis au lecteur de textes polémiques recourent à un vocabulaire marquant l'objectif : l'introduction d'un libelle affirme ainsi que la publication qui suit est imprimée « affin qu'esveillez \& faicts sages par l'exemple d'autruy, [...] [les Catholicques] s'efforcent d'observer diligemment la saincte loy \& volonté de Dieu ${ }^{63}$ »; une autre « afin que par ces signes nous soyons admonestez de nous comporter plus humblement ${ }^{64}$ ". La rhétorique délibérative vient renforcer les genres judiciaire et épidictique par des propos anagogiques. La portée de tels passages au sein des avis au lecteur des libellistes est entièrement polémique: les recommandations en faveur d'une bonne lecture ne visent pas à former un lecteur érudit mais à faire agir un catholique zélé. L'imprimeur de la Metamorphose d'Henry de Bourbon précise ainsi qu'il a conçu ce libelle « afin que chacun sçache [...] que [...] ledict Henry de Navarre, ny de droict ny de faict, ne peut rien pretendre à la Couronne de France, \& à fin aussi que chacun s'oppose plus hardiment \& plus vaillamment à c'est avorton de la maison de Bourbon ${ }^{65}$. » La préface du libelle construit une « performativité de l'œuvre ${ }^{66}$ » et unit les lecteurs au sein d'une communauté d'action découlant du texte introduit ${ }^{67}$.

Les marges du texte polémique constituent le second lieu de déploiement de texte d'escorte prisé par les libellistes. À plusieurs égards, elles fonctionnent en système avec les pièces liminaires. Ainsi, l'avis au lecteur de la Lettre escrite à Madame de Tinteville apporte une précision à propos des notes marginales qui ornent le texte publié : « Je vous laisse juge par ceste lecture, à laquelle j'adjouste peu de mots en marge pour esclaircir ce qui pourroit apporter doute sans changer au cours de ladicte missive une seule lettre. » Les points de partage entre marginalia et avis au lecteur sont nombreux : une fonction didactico-polémique, le recours à la technique du texte « publié » qui prouve l'idée défendue et légitime de la diffusion d'un imprimé, et, enfin, une posture raisonnée et une rhétorique judiciaire ambigües qui affirment laisser le jugement au lecteur tout en le guidant tellement dans sa réflexion qu'une seule conclusion peut émerger de sa lecture. Les marginalia achèvent la construction du scandale en désignant les éléments saillants du texte publié et en expliquant, en quelques mots, comment ils doivent être interprétés.

Certains commentaires marginaux sont ouvertement polémiques. Ainsi, un des passages de la Lettre escrite à Madame de Tinteville est qualifié de «blaspheme » en marge ${ }^{68}$. Les marginalia de la Metamorphose d'Henry de Bourbon sont composées d'accusations plus explicites encore : «Il a bien changé de langage ${ }^{69}$ » ; «Ils ont bien faussé leurs promesses ${ }^{70}$. » Les pièces liminaires et les marges fonctionnent en système afin d'accuser l'adversaire : l'avis au lecteur mentionne

63 Discours veritable du martyre de deux prebstres \& deux laycs, op. cit., p. 4. Nos italiques.

64 Ibid., p. 3. Nos italiques.

65 Metamorphose d'Henry de Bourbon, op. cit., p. 4. Nos italiques.

66 Patrick Marot, « Pour une poétique historique des textes liminaires », art. cit., p. 8.

67 Sur le caractère fédérateur du scandale et de la rumeur, voir Émilie Dosquet et François-Xavier Petit, « Faire scandale », art. cit., p. 155-156; N Nathalie Heinich, «L'art du scandale », art. cit.; Max Gluckman, « Gossip and Scandal », art. cit. Sur la notion de textes d'action, voir Christian Jouhaud, Mazarinades. La Fronde des mots, Paris, Aubier, 1985, p. 37-38; Id., « Les libelles en France au XVIIe siècle : action et publication », Cahiers d'histoire. Revue d'histoire critique, 90-91, 2003, p. 33-45, [En ligne] https:// journals.openedition.org/chrhc/1443 [consulté le 28/10/2021].

68 Lettre escrite à madame de Tinteville, op. cit., p. 5.

69 Metamorphose d'Henry de Bourbon, op. cit., p. 6.

70 Ibid., p. 14. 
le grief principal - en l'occurrence, la versatilité -, les marginalia identifient les preuves et en formulent les conclusions spécifiques. Prenant tous deux appui sur un ethos didactique, la rhétorique judiciaire ${ }^{71}$ et le blâme constituent les deux facettes de l'accusation de l'adversaire dans les marges du libelle. L'un des marginalia du libelle interpelle le lecteur par une question rhétorique ouvertement polémique ${ }^{72}$ : «Si Henry de Bourbon n'a pas esté constant en la foy catholique, [s]i aiant baillé la parolle d'estre bon catholique à ceux icy, pensez vous qu'il le sera jamais ${ }^{73}$ ? » Les libellistes exploitent les possibilités discursives offertes par la brièveté et le grand nombre des gloses marginales pour y distiller de violents blâmes au sein d'une rhétorique globalement judiciaire. La Metamorphose d'Henry de Bourbon l'illustre à nouveau: « Henry de Bourbon enfant d'ire \& non de Dieu ${ }^{74}$ »; « Henry de Bourbon capitaine des heretiques de France $^{75} \gg$.

Tout à l'inverse, certains marginalia sont composés d'un vocabulaire mesuré : identifiant des personnages ${ }^{76}$, rappelant le contexte ${ }^{77}$, précisant la nature du texte publié ${ }^{78}$, ils semblent poursuivre des objectifs purement didactiques. L'ethos ainsi construit constitue alors une preuve qui conforte l'ensemble de la publication. Les liens entre didactique et polémique sont encore plus étroits quand la marge accuse sous couvert d'aider le lecteur à comprendre les enjeux par une paraphrase du contenu. Cette stratégie rhétorique peut s'observer dans la Metamorphose d'Henry de Bourbon : "Sa Saincteté excuse sa faute premiere pour cause de son bas aage, mais [ce] qu'il a faict depuys est inexcusable ${ }^{79} »$. La première partie du marginalium est objective, la seconde non : l'interprétation polémique des faits est dissimulée par une apparence de neutralité et de pédagogie. La marge se pare d'une façade descriptive pour mieux masquer qu'elle est fondamentalement polémique : "Benignité du S. Siege apostolique envers ledict Henry, mal recogneue par iceluy ${ }^{80}$ »; « Bons maistres d'Henry de Bourbon, lesquels il n'a suivi ${ }^{81} »$. Par le recours à la phrase nominale, les polémistes utilisent la syntaxe traditionnelle de la glose marginale pour parer d'une neutralité d'apparence une accusation pourtant évidente. Le marginalium est le lieu d'un contraste saisissant entre une syntaxe feignant l'objectivité et un vocabulaire extrêmement péjoratif voire agressif : « Grande faintise de Henry de Bourbon, ou s'il n'y a poinct eu de faintise, grande desloyauté \& perfidie d'iceluy ${ }^{82}$ » « « CEuvres signalees de Henry de Bourbon par lesquelles il ne merite d'estre couronné Roy de France, ains tous les catholiques s'y doivent opposer, \& luy courir sus, comme à un enfant de $\operatorname{Satan}^{83}$ ». Quelques irrégularités dans l'emploi systématique de la phrase nominale tendent à indiquer une rapidité dans la composition du libelle: "Difficulté grande de convertir un heretique \& partant ne croyez pas qu'Henry relaps en heresie se soit couvert à bon escient ${ }^{84}$ ». Quel que soit le registre retenu, la marge recourt à un raisonnement de type axiologique, dont la

71 «L'accusation procède par accumulation des accusations partielles qui rendent vraisemblable la charge principale. » (Tatiana Debbagi Baranova, "La vérité et les stratégies d'accréditation du discours », art. cit.)

72 «L'accumulation de questions oratoires reformulant inlassablement la même idée presse l'auditeur de toutes parts et crée un effet de sidération morale. » (Marion Gauthier, Scandale et indignation, op. cit., p. 302.)

73 Metamorphose d'Henry de Bourbon, op. cit., p. 5-6.

74 Ibid., p. 30.

75 Ibid., p. 34.

76 Lettre escrite à madame de Tinteville, op. cit., p. 6.

77 Ibid., p. 7.

78 Metamorphose d'Henry de Bourbon, op. cit., p. 7 et 16.

79 Ibid., p. 8.

80 Ibid., p. 11.

81 Ibid., p. 31.

82 Ibid., p. 13.

83 Ibid., p. 34-35.

84 Ibid., p. 19. 
propriété est de produire une codification, une normalisation et une objectivation de la réalité 85 . La dénomination des fautes et des travers des adversaires est essentielle à la polémique car elle lui permet d'identifier clairement les motifs de l'indignation qu'elle cherche à susciter ${ }^{86}$. La construction du réel est une opération clef de la fabrication du scandale : l'accusation ne peut pas se contenter d'extraire du champ événementiel les faits à même de constituer des preuves, elle doit aussi les qualifier, c'est-à-dire les pointer du doigt, expliciter leur importance et annoncer par anticipation leurs conséquences ${ }^{87}$. La marge du texte, aux propriétés bien connues de captatio, est le lieu idéal pour procéder à cette indispensable désignation du scandale ${ }^{88}$.

D'un point de vue formel, les polémistes exploitent les marges du texte avec créativité et liberté. Ainsi, quand le paratexte est dense, l'imprimeur de la Metamorphose d'Henry de Bourbon recourt à un système de numérotation des marginalia proche de nos notes de bas de pages contemporaines : une lettre en exposant dans le texte "publié » renvoie à la glose correspondante en marge ${ }^{89}$. L'utilisation de formes élaborées d'annotations marginales est davantage systématique dans une missive « interceptée » et « publiée » par la Ligue parisienne sous le nom de Coppie d'une lettre du cardinal de Vendosme à Monsieur de Luxembourg envoyee de Rome. Le texte est reproduit sur toute la largeur de la page : les notes ne se trouvent pas dans la marge mais après la lettre et sont reliées au texte principal par un système de nombres en exposant. Cette pratique d'annotation, proche de nos notes de fin contemporaines, prend le nom d'« apostilles et remarques ${ }^{90} »$ dans le libelle. Pour fabriquer le scandale, le texte « publié » devient « édité » : les libellistes poursuivent leur effort de construction du réel par un travail de commentaire en profondeur - les apostilles occupent plus d'espace dans le libelle que le texte de la lettre - désignant avec systématisme les faits propres à susciter l'indignation.

Les marginalia sont exploités selon des pratiques encore plus exceptionnelles dans la Lettre intercepte du Prince d'Oranges au duc d'Alençon ${ }^{91}$. La page est divisée en deux colonnes en miroir : dans la première, la lettre est divisée en paragraphes comportant trois ou quatre chiffres arabes en exposant renvoyant aux commentaires en regard dans la seconde. La lettre est reproduite dans une police plus grande que celle des commentaires qui, nettement plus denses, sont répartis en quarante-huit blocs-textes resserrés et débordant parfois sur la colonne de la lettre ${ }^{92}$. Dans une autre édition, les pages ne sont pas divisées en colonnes : le texte de la lettre est reproduit sur les pages paires, en regard des commentaires imprimés sur les pages impaires ${ }^{93}$. Dans les deux cas, la fonction de ces commentaires est clairement énoncée par le sous-titre du libelle : ils sont rédigés " pour ouvrir les jeulx [sic] aux bons subjectz ». Leur contenu, identique d'une édition à l'autre, confirme que la marge constitue le lieu où le scandale se fabrique par une construction du réel.

85 Marc Angenot, Dialogue de sourds. Traité de rhétorique antilogique, Paris, Mille et une Nuits, 2008, p. 240-245.

86 Ruth Amossy, Apologie de la polémique, op. cit., p. 160-164.

87 Luc Boltanski et Élisabeth Claverie, «Du monde social en tant que scène d'un procès », dans Luc Boltanski et al. (dir.), Affaires, scandales et grandes causes, op. cit., p. 438-442.

88 Grégoire Menu, «La Cour dans les marges, en marge de la cour : usages rhétoriques et politiques des notes marginales dans La Cour sainte de Nicolas Caussin », dans Yohann Deguin et Marine Roussillon (dir.), Depuis les marges, op. cit. ; Patrick Marot, « Pour une poétique historique des textes liminaires », art. cit.

89 Metamorphose d'Henry de Bourbon, op. cit., p. 21.

90 Coppie d'une lettre du cardinal de Vendosme à Monsieur de Luxembourg envoyee de Rome, Paris, Nicolas Nivelle et Rolin Thierry, 1590, p. 8.

91 D'après l'Universal Short Title Catalogue, ce texte paru sans adresse provient des presses montoises de Roger Velpius et sont rédigés par Christophe d'Assonleville.

92 Lettre intercepte du prince d'Oranges au duc d'Alençon, op. cit., p. [10], [14] et [15].

93 Cette édition est consultable en ligne sur https://biblio.umons.ac.be/public/bv/imp110031g/ [consulté le 28/10/2021]. 
Les marginalia s'emploient en effet à désigner les différents éléments convergeant vers le grief principal que la publication entend démontrer : la duplicité du prince d'Orange, chef des révoltés protestants dans les Pays-Bas. Exploitant ces images topiques du masque et du voile, le libelliste dénonce l'ennemi des Habsbourg ${ }^{94}$ en des termes très proches de ceux avec lesquels la Ligue catholique française diffamera le roi Henri III une dizaine d'années plus $\operatorname{tard}^{95}$. Les explications que l'avis au lecteur du libelle fournit au sujet des marginalia constituent une parfaite synthèse de leur rôle dans la fabrique du scandale:

Je y ay, comme desireux de ton bien, adjousté quelques appostilles pour grossierement faire entendre aux plus ignorans ${ }^{96}$ les desseings de ce meschant homme. Mais arreste toy sur le texte de la lettre, qui est d'estat, \& de sustance [sic], \& par laquelle tu congnoistras l'inclination, qu'il à a [sic] la ruine \& totale destruction de ce pauvre \& affligé pais.

Le caractère indissociable du texte et de sa marge est explicité par l'avis au lecteur de ce libelle. Les « appostilles » ont pour objectif de « faire entendre », c'est-à-dire comprendre - leur fonction est didactique -, la lettre de faire « congnoistre », c'est-à-dire identifier, avoir la révélation et la certitude, reconnaître au sens juridique - sa fonction est argumentative. Les deux éléments participent à une construction du réel mêlant didactique et polémique sur fond de posture raisonnée et cadrée : telles sont les conditions de la fabrication du scandale par le paratexte du libelle.

La nature publique du scandale représente à la fois un défi et une opportunité pour les libellistes. Leurs usages rhétoriques du paratexte effectuent deux actions conjointes : ils évitent l'accusation de scandale au sens politique et le fabriquent puis le rejettent, au sens évangélique, sur leurs adversaires. Permettant, en amont, de justifier la publication et, en aval, d'appeler à l'action ceux qui le lisent, le paratexte est un outil indispensable au sein de toutes les étapes de la construction du réel résultant de la fabrique du scandale par le libelle. Unissant comment et pourquoi, didactique et polémique, légitimation et accusation, le paratexte noue les fils du scandale. La publication de textes apocryphes et assortis d'une pièce liminaire, jouant sur la pluriauctorialité fictive qui en résulte et exploitant la liberté dans la disposition du texte ainsi que de ses gloses marginales sur la page, illustrent la créativité des polémistes dans la fabrique du scandale par le paratexte des libelles. Les libellistes exploitent cette notion de scandale dans une ambivalence assumée et presque ludique entre ses deux acceptions. Friands de glissements, ils l'utilisent pour tisser des liens entre différentes sphères, instrumentalisant à des fins politiques les motifs religieux de l'indignation et du scandale. En matière de dépassement des frontières, on ne peut manquer de relever que les textes étudiés font état de pratiques discursives similaires sinon concordantes entre la France ligueuse et les Pays-Bas habsbourgeois.

94 Guillaume d'Orange y est qualifié de « double Renard » (Lettre intercepte du prince d'Oranges au duc d'Alençon, op. cit., p. [6]), de « si meschant \& desloyal dragon » (ibid., p. [7]) et de « faulx Hypocrite» (ibid., p. [6]). Voir Monica Stensland, Habsburg communication in the Dutch revolt, op. cit., p. 92-93; Ead., " Not as bad as all that. The strategies and effectiveness of Loyalist propaganda in the early years of Alexander Farnese's governorship », Dutch Crossing, 21, 2007, p. 94, [En ligne] https://www. tandfonline.com/doi/abs/10.1080/03096564.2007.11730893 [consulté le 28/10/2021].

95 Sur ce vocabulaire commun de la duplicité entre les deux corps, voir Alexandre Goderniaux, "Le "voile commun à tous meschans" », art. cit.

96 Ce terme peut faire écho à la tradition thomiste du « scandale des petits ». Voir Anne-Pascale Pouey-Mounou, Panurge comme lard en pois, op. cit., p. 77 et 97-98. Le médisant représente un danger avant tout pour les faibles: Tatiana Debbagi Baranova, « Les guerres de Religion : guerres de médisance? », art. cit., p. 467 et 477-478. 
Alexandre Goderniaux

Boursier FRESH (ULiège, Unité de Recherches Transitions)

Retrouvez la version en ligne gratuite et ses contenus additionnels

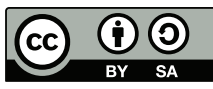


